\title{
Disformal transformation of physical quantities associated with relativistic stars
}

\author{
Masato Minamitsuji ${ }^{1}$ \\ ${ }^{1}$ Centro de Astrofísica e Gravitação - CENTRA, \\ Departamento de Física, Instituto Superior Técnico - IST, \\ Universidade de Lisboa - UL, Av. Rovisco Pais 1, 1049-001 Lisboa, Portugal
}

\begin{abstract}
We investigate how physical quantities associated with relativistic stars in the Jordan and Einstein frames are related by the generalized disformal transformations constructed by the scalar and vector fields within the slow-rotation approximation. We consider the most general scalar disformal transformation constructed by the scalar field, and by the vector field without and with the $U(1)$ gauge symmetry, respectively. At the zeroth order of the slow-rotation approximation, by imposing that both the metrics of the Jordan and Einstein frames are asymptotically flat, we show that the Arnowitt-Deser-Misner mass is frame-invariant. At the first order of the slow-rotation approximation, we discuss the disformal transformations of the frame-dragging function, angular velocity, angular momentum, and moment of inertia of the star. We show that the angular velocity of the star is frame-invariant in all the cases. While the angular momentum and moment of inertia are invariant under the scalar disformal transformation, they are not under the vector disformal transformation without and with the $U(1)$ gauge symmetry.
\end{abstract}

\section{INTRODUCTION}

While general relativity (GR) has passed all the experimental tests in weak gravity regimes [1], with the latest measurements of gravitational waves $[2-4]$ and black hole shadows $[5,6]$, a new frontier for testing GR in strongfield regimes has opened. Black holes are the most fundamental objects in theories of gravitation. While many modified theories of gravitation share the same black hole solutions with GR $[7,8]$, other theories also admit black solutions with the nontrivial profiles of the non-metric field.

Relativistic stars as neutron and quark stars $[9,10]$ are known to be the second most compact objects in the Universe. Scalar-tensor theories of gravitation with nonminimal coupling to the matter sector admit large deviations from GR through the mechanism called spontaneous scalarization [11-19], triggered by the conformal coupling to the matter sector

$$
\tilde{g}_{\mu \nu}=\mathcal{C}(\phi) g_{\mu \nu}
$$

where the Greek induces $(\mu, \nu, \cdots)$ run the fourdimensional spacetime and $\mathcal{C}(\phi)(>0)$ is the regular function of the scalar field $\phi$. In this paper, we call the frames with the metrics $g_{\mu \nu}$ and $\tilde{g}_{\mu \nu}$ the Einstein and Jordan frames, respectively, where the action of the matter sector $\Psi_{m}$ is given by

$$
S \supset S_{m}\left[\tilde{g}_{\mu \nu}, \Psi_{m}\right]:=\int d^{4} x \sqrt{-\tilde{g}} \mathcal{L}_{m}\left[\tilde{g}_{\mu \nu}, \Psi_{m}\right] .
$$

The energy-momentum tensors of matter in the Jordan and Einstein frames, $\tilde{T}_{(m)}^{\mu \nu}$ and $T_{(m)}^{\mu \nu}$, respectively, are related by

$$
T_{(m)}^{\mu \nu}:=\frac{2}{\sqrt{-g}} \frac{\delta\left(\sqrt{-\tilde{g}} \mathcal{L}_{m}\right)}{\delta g_{\mu \nu}}=\sqrt{\frac{\tilde{g}}{g}} \frac{\delta \tilde{g}_{\alpha \beta}}{\delta g_{\mu \nu}} \tilde{T}_{(m)}^{\alpha \beta},
$$

with

$$
\tilde{T}_{(m)}^{\mu \nu}:=\frac{2}{\sqrt{-\tilde{g}}} \frac{\delta\left(\sqrt{-\tilde{g}} \mathcal{L}_{m}\right)}{\delta \tilde{g}_{\mu \nu}} .
$$

Ref. [20] considered spontaneous scalarization in the model with the scalar disformal coupling

$$
\tilde{g}_{\mu \nu}=\mathcal{C}(\phi)\left(g_{\mu \nu}+\mathcal{B}(\phi) \phi_{\mu} \phi_{\nu}\right) \text {, }
$$

where $\mathcal{B}(\phi)$ is the regular function of $\phi, \phi_{\mu}:=\nabla_{\mu} \phi$ represents the covariant derivative of the scalar field, and the canonical kinetic term of the scalar field is defined by

$$
\mathcal{X}:=-\frac{1}{2} g^{\mu \nu} \phi_{\mu} \phi_{\nu}
$$

The extension of spontaneous scalarization to the vector field $A_{\mu}$, i.e., spontaneous vectorization, has been investigated in Refs. [21-26]. Ref. [23] considered a model with nonminimal coupling to the Ricci tensor $R^{\mu \nu} A_{\mu} A_{\nu}$ and the Ricci scalar $R g^{\mu \nu} A_{\mu} A_{\nu}$, and Ref. [25] studied a model with nonminimal coupling to the Einstein tensor $G^{\mu \nu} A_{\mu} A_{\nu}$ in the generalized Proca theories [27, 28]. Ref. [21] considered the vector conformal coupling

$$
\tilde{g}_{\mu \nu}=\mathcal{D}(\mathcal{Y}) g_{\mu \nu},
$$

where $\mathcal{D}(\mathcal{Y})$ is the regular function of the spacetime norm of the vector field,

$$
\mathcal{Y}:=-\frac{1}{2} g^{\mu \nu} A_{\mu} A_{\nu} .
$$

Ref. $[24,26]$ argued the case of the vector disformal coupling

$$
\tilde{g}_{\mu \nu}=g_{\mu \nu}+\mathcal{E}(\mathcal{Y}) A_{\mu} A_{\nu}
$$

where $\mathcal{E}(\mathcal{Y})$ is the regular function of $\mathcal{Y}$.

However, before exploring more generalized models of spontaneous scalarization and vectorization, as well as other models such as spontaneous spinorization [26, 29], we have to clarify the dependence of physical quantities associated with relativistic stars on the choice of the frames. Although the physical frame in these models is the Jordan frame where the scalar or vector field is not directly coupled to the matter sector, in many 
cases it is more convenient to evaluate the same quantities in the Einstein frame. In such a case, in order to translate the quantities defined in the Einstein frame to those in the Jordan frame, we have to clarify the relation of them defined in both the frames beforehand. Since the frame transformation mathematically corresponds to the change of the units or the measures, we naively expect that the observationally relevant quantities should be independent of the choice of the frames. However, the frame dependence of physical quantities is rather a subtle issue and should be clarified in each of the different cases. For instance, in the context of cosmology in scalar-tensor theories, the frame-invariance of cosmological redshift, distance-duality relation, scalar and tensor cosmological perturbations, anisotropy of Cosmic Microwave Backgrounds, and so on have been argued under the conformal and disformal transformations (see e.g., Refs. [30-38]).

In the case of the systems associated with relativistic stars, to our knowledge, the frame-invariance of the observables has been less studied. In the case of scalartensor theories with the disformal coupling (5) including the ordinary conformal coupling case (1). Ref. [20] showed that the Arnowitt-Deser-Misner (ADM) mass, frame-dragging function, angular velocity, angular momentum and moment of inertia of the star are frameinvariant with the context of the slow-rotation approximation $[39,40]$.

The purpose of this paper is to extend the previous work [20] to more general classes of the disformal transformation constructed by the scalar and vector fields. More specifically, we will focus on the following three classes of the disformal transformations;

(1): most general disformal transformation constructed by the scalar field and its first-order derivative;

$$
\tilde{g}_{\mu \nu}=\mathcal{C}(\mathcal{X}, \phi)\left(g_{\mu \nu}+\mathcal{B}(\mathcal{X}, \phi) \phi_{\mu} \phi_{\nu}\right)
$$

where $\mathcal{C}$ and $\mathcal{B}$ are the regular functions of $\mathcal{X}$ and $\phi$ defined in Eq. (6) (see Sec. III).

(2): disformal transformation constructed by the vector field without the $U(1)$ gauge symmetry;

$$
\tilde{g}_{\mu \nu}=\mathcal{D}(\mathcal{Y})\left(g_{\mu \nu}+\mathcal{E}(\mathcal{Y}) A_{\mu} A_{\nu}\right)
$$

which generalizes the pure conformal and disformal transformations (7) and (9) (see Sec. IV).

(3): disformal transformation constructed by the vector field with the $U(1)$ gauge symmetry;

$$
\tilde{g}_{\mu \nu}=\mathcal{V}(\mathcal{F}, \mathcal{G})\left(g_{\mu \nu}+\mathcal{W}(\mathcal{F}, \mathcal{G}) g^{\alpha \beta} F_{\mu \alpha} F_{\nu \beta}\right)
$$

where $\mathcal{V}$ and $\mathcal{W}$ are the regular functions of

$$
\begin{aligned}
\mathcal{F} & :=g^{\mu \alpha} g^{\nu \beta} F_{\mu \nu} F_{\alpha \beta}, \\
\mathcal{G} & :=g^{\mu \nu} g^{\alpha \beta} g^{\rho \sigma} g^{\gamma \delta} F_{\mu \alpha} F_{\rho \beta} F_{\sigma \gamma} F_{\nu \delta}
\end{aligned}
$$

with the $U(1)$ gauge field strength

$$
F_{\mu \nu}:=\partial_{\mu} A_{\nu}-\partial_{\nu} A_{\mu}
$$

(see Sec. V).

The invertible scalar disformal transformation (10) can frame the degenerate higher-order scalar-tensor theories [41-44], which are known as the most general singlefield scalar-tensor theories without Ostrogradsky instabilities [45]. The invertible vector disformal transformation without the $U(1)$ gauge symmetry (11) can map the generalized Proca theories [27, 28, 46], which are known as the most general vector-tensor theories with the second-order equations of motion, to the extended vector-tensor theories without Ostrogradsky instabilities constructed in Ref. [47]. Similarly, the invertible vector disformal transformation (12) constitutes the most general frame transformation with the vector field under the $U(1)$ gauge symmetry, which maps the Einstein-Maxwell theory to the degenerate higher-order vector-tensor theories with the $U(1)$ gauge symmetry and without Ostrogradsky instabilities [48, 49].

This paper is constructed as follows: In Sec. II, we introduce the slowly rotating star supported by the perfect fluid and the spacetime metric in the slow-rotation approximation. In Sec. III, we discuss how physical quantities associated with relativistic stars are transformed under the disformal transformation constructed by the scalar field (10). In Secs. IV and V, we study the same subject in the case of the disformal transformations constructed by the vector field without and with the $U(1)$ symmetry, Eqs. (11) and (12), respectively. The last Sec. VI is devoted to giving a brief summary and conclusion.

\section{SLOWLY ROTATING STARS}

We consider the rigidly rotating star with the constant angular velocity $\Omega$, and adopt the slow-rotation approximation [39, 40], where the variables of the metric and other degrees of freedom are expanded with respect to the small parameter $\epsilon(\ll 1)$, which is of the order of the dimensionless angular speed of the star $\Omega \tilde{\mathcal{R}}(\ll 1)$, with $\tilde{\mathcal{R}}$ being the radius of the star. At $\mathcal{O}\left(\epsilon^{0}\right)$, the metric and the scalar or vector field equations of motion coincide with those in the static and spherically symmetric spacetime. At $\mathcal{O}\left(\epsilon^{1}\right)$, the leading-order corrections due to the slow rotation of the star, which appear in the frame-dragging component of the spacetime metric $g_{t \varphi}$ and the azimuthal component of the vector field $A_{\varphi}$, are obtained on top of the static and spherically symmetric backgrounds.

Up to $\mathcal{O}\left(\epsilon^{1}\right)$, the metric inside and outside of the star can be expressed as

$$
\begin{aligned}
g_{\mu \nu} d x^{\mu} d x^{\nu} & =-f(r) d t^{2}+\frac{d r^{2}}{h(r)}+r^{2}\left(d \theta^{2}+\sin ^{2} \theta d \varphi^{2}\right) \\
& +2 \epsilon r^{2}(\omega(r)-\Omega) \sin ^{2} \theta d t d \varphi+\mathcal{O}\left(\epsilon^{2}\right)
\end{aligned}
$$


where $t, r$, and $(\theta, \varphi)$ represent the time, radial, and angular coordinates, respectively, $f(r)$ and $h(r)$ are the functions of $r$, and $\omega(r)$ and $\Omega$ represent the framedragging function and the angular velocity of the star. $f(r), h(r)$, and $\omega(r)$ are continuous across the surface of the star.

We impose the asymptotic flatness of the Einsteinframe metric $g_{\mu \nu}$ in the limit of $r \rightarrow \infty$, which is given by

$$
\begin{aligned}
& f(r \rightarrow \infty) \rightarrow \text { const }, \\
& h(r \rightarrow \infty) \rightarrow 1, \\
& \omega(r \rightarrow \infty) \rightarrow \Omega .
\end{aligned}
$$

We assume that the Jordan-frame metric $\tilde{g}_{\mu \nu}$, Eq. (18), satisfies the circularity conditions [50-52]; if the two commuting Killing vector fields in the stationary and axisymmetric spacetime $\xi^{\mu}=(\partial / \partial t)^{\mu}$ and $\sigma^{\mu}=(\partial / \partial \varphi)^{\mu}$ satisfy

$$
\sigma_{[\mu} \xi_{\nu} \tilde{\nabla}_{\alpha} \xi_{\beta]}=0, \quad \sigma_{[\mu} \xi_{\nu} \tilde{\nabla}_{\alpha} \sigma_{\beta]}=0,
$$

where $\tilde{\nabla}_{\mu}$ represents the covariant derivative associated with the metric $\tilde{g}_{\mu \nu}$, then the spacetime metric reduces to the block-diagonal form with $\tilde{g}_{t \tilde{r}}=\tilde{g}_{t \theta}=\tilde{g}_{\tilde{r} \varphi}=\tilde{g}_{\theta \varphi}=0$, which in the slow-rotation approximation reduces to the form [53];

$$
\begin{aligned}
\tilde{g}_{\mu \nu} d x^{\mu} d x^{\nu} & =-\tilde{f}(\tilde{r}) d t^{2}+\frac{d \tilde{r}^{2}}{\tilde{h}(\tilde{r})}+\tilde{r}^{2}\left(d \theta^{2}+\sin ^{2} \theta d \varphi^{2}\right) \\
& +2 \epsilon \tilde{r}^{2} \sin ^{2} \theta(\tilde{\omega}(\tilde{r})-\tilde{\Omega}) d t d \varphi \\
& +\mathcal{O}\left(\epsilon^{2}\right)
\end{aligned}
$$

where the new radial coordinate $\tilde{r}(r)$, and the new metric functions $\tilde{f}(\tilde{r}), \tilde{h}(\tilde{r})$, and $\tilde{\omega}(\tilde{r})$ are related to the original ones by the direct comparison with Eqs. (10), (11), and (12). This requirement is automatically satisfied for the disformal transformations (10) and (12), but restricts the form of the vector field in the case of the vector disformal transformation without the $U(1)$ gauge symmetry (11) (see Sec. IV). We assume the circularity conditions (17) for several reasons. First, in order for the physical quantities to be frame-invariant, we expect that the metrics in the Jordan and Einstein frames should share the same form, and that there always exist counterparts of the metric and matter variables in both the frames. Second, imposing the circularity conditions (17) simplifies the computations. In the case of black holes, it was recently argued in Ref. [54] that in the effective theory description of non-GR gravitational theories the spacetime of stationary, axisymmetric, and asymptotically flat solutions has to be circular, when the solutions are constructed perturbatively from the GR solutions. The similar properties may exist also for the solutions of relativistic stars constructed perturbatively from the GR ones.

On the other hand, the physical consequences of the violation of the circularity conditions on the dynamical quantities and their frame transformations are worth being investigated and for now will be left for future work.
We expect that no physical pathology associated with the violation of the circularity conditions would arise, such as instabilities. However, the noncircular spacetime around the star would modify the motion of photons and particles in the vicinity of the stars, and affect the measurements of pulsars and gravitational waves. Thus, if no peculiar deviation is detected in the future, the deviations from the circular spacetimes would be significantly constrained.

Here, we require that $\tilde{r}(r)$ is the monotonically increasing function of $r$, and impose the asymptotic flatness of $\tilde{g}_{\mu \nu}$ in the limit of $\tilde{r} \rightarrow \infty$

$$
\begin{aligned}
& \tilde{f}(\tilde{r} \rightarrow \infty) \rightarrow \text { const }>0, \\
& \tilde{h}(\tilde{r} \rightarrow \infty) \rightarrow 1, \\
& \tilde{\omega}(\tilde{r} \rightarrow \infty) \rightarrow \tilde{\Omega} .
\end{aligned}
$$

We consider the star composed of matter whose energy-momentum tensor in the Einstein frame is given in the perfect fluid form

$$
\begin{aligned}
T_{(m) \mu \nu} & =\rho u_{\mu} u_{\nu}+p_{r} k_{\mu} k_{\nu} \\
& +p_{t}\left(g_{\mu \nu}+u_{\mu} u_{\nu}-k_{\mu} k_{\nu}\right),
\end{aligned}
$$

where the components of the vector fields, up to $\mathcal{O}\left(\epsilon^{1}\right)$, are defined by

$$
\begin{aligned}
& u_{\mu}=\left(-\sqrt{-g_{t t}}, 0,0, \epsilon \frac{r^{2} \omega \sin ^{2} \theta}{\sqrt{-g_{t t}}}\right)+\mathcal{O}\left(\epsilon^{2}\right), \\
& k_{\mu}=\left(0, \sqrt{g_{r r}}, 0,0\right)+\mathcal{O}\left(\epsilon^{2}\right) .
\end{aligned}
$$

Correspondingly, the energy-momentum tensor of matter in the Jordan frame is also assumed to be given by the perfect fluid form Eqs. (20)-(21) with the replacement of all the quantities to those with 'tilde', which are related through Eq. (3) with Eqs. (10), (11), and (12). The surface of the star is fixed by the condition $\tilde{p}_{r}(\tilde{\mathcal{R}})=0$. As we will see in Secs. III-V , in general the condition $\tilde{p}_{r}(\tilde{\mathcal{R}})=0$ does not imply that $p_{r}(\mathcal{R})=0$ with $\tilde{\mathcal{R}}=\tilde{\mathcal{R}}(\mathcal{R})$, and the concrete study in each case of the disformal transformations, Eqs. (10), (11), and (12), is necessary.

At $\mathcal{O}\left(\epsilon^{0}\right)$, the mass functions in both frames, $M(r)$ and $\tilde{M}(\tilde{r})$, are defined by

$$
\begin{aligned}
M(r) & =\frac{r}{2}(1-h(r)), \\
\tilde{M}(\tilde{r}) & =\frac{\tilde{r}}{2}(1-\tilde{h}(\tilde{r})),
\end{aligned}
$$

whose limits to $r \rightarrow \infty$ and $\tilde{r} \rightarrow \infty$ provide the ADM masses, respectively,

$$
\begin{aligned}
M_{\mathrm{ADM}} & :=M(r \rightarrow \infty), \\
\tilde{M}_{\mathrm{ADM}} & :=\tilde{M}(\tilde{r} \rightarrow \infty) .
\end{aligned}
$$

Up to $\mathcal{O}\left(\epsilon^{1}\right)$, the angular momenta and moments of in- 
ertia in both frames are given, respectively, by

$$
\begin{aligned}
& \mathcal{J}:=\int d r d \theta d \varphi r^{2} \sin \theta \sqrt{\frac{f}{h}} T_{(m)}{ }^{t} \varphi, \\
& \tilde{\mathcal{J}}:=\int d \tilde{r} d \theta d \varphi \tilde{r}^{2} \sin \theta \sqrt{\frac{\tilde{f}}{\tilde{h}}} \tilde{T}_{(m)}{ }^{t} \varphi
\end{aligned}
$$

and

$$
\begin{aligned}
\mathcal{I} & :=\frac{\mathcal{J}}{\Omega}, \\
\tilde{\mathcal{I}} & :=\frac{\tilde{\mathcal{J}}}{\tilde{\Omega}} .
\end{aligned}
$$

\section{THE CASE OF THE SCALAR DISFORMAL TRANSFORMATION}

In this section, we consider the scalar-tensor theories

$$
S=S_{g}\left[g_{\mu \nu}, \phi\right]+S_{m}\left[\tilde{g}_{\mu \nu}, \Psi_{m}\right],
$$

where the matter action is given by Eq. (2) and the relation between the two metrics is given by Eq. (10).

In the slow-rotation approximation, we assume the spacetime metric (15) and the scalar field given by

$$
\begin{aligned}
& \phi=\phi_{0}(r)+\mathcal{O}\left(\epsilon^{2}\right), \\
& \mathcal{X}=\mathcal{X}_{0}(r)+\mathcal{O}\left(\epsilon^{2}\right):=-\frac{h(r) \phi_{0}^{\prime}(r)^{2}}{2}+\mathcal{O}\left(\epsilon^{2}\right) .
\end{aligned}
$$

The functions $\mathcal{C}$ and $\mathcal{B}$ can be expanded as

$$
\begin{aligned}
\mathcal{C} & =\mathcal{C}_{0}(r)+\mathcal{O}\left(\epsilon^{2}\right) \\
& :=\mathcal{C}\left(\mathcal{X}_{0}(r), \phi_{0}(r)\right)+\mathcal{O}\left(\epsilon^{2}\right), \\
\mathcal{B} & =\mathcal{B}_{0}(r)+\mathcal{O}\left(\epsilon^{2}\right) \\
& :=\mathcal{B}\left(\mathcal{X}_{0}(r), \phi_{0}(r)\right)+\mathcal{O}\left(\epsilon^{2}\right), \\
\mathcal{C}_{0 \mathcal{X}}(r) & :=\mathcal{C}, \mathcal{X}\left(\mathcal{X}_{0}(r), \phi_{0}(r)\right), \\
\mathcal{B}_{0 \mathcal{X}}(r) & :=\mathcal{B}, \mathcal{X}\left(\mathcal{X}_{0}(r), \phi_{0}(r)\right),
\end{aligned}
$$

where $\mathcal{C}, \mathcal{X}:=\partial \mathcal{C} / \partial \mathcal{X}$ and $\mathcal{B}, \mathcal{X}:=\partial \mathcal{B} / \partial \mathcal{X}$. Comparing frames relates the radial coordinates and the metric components in the Jordan and Einstein frames, respectively, as

$$
\tilde{r}:=\sqrt{\mathcal{C}_{0}(r)} r
$$

and

$$
\begin{aligned}
\tilde{f}(\tilde{r}) & =\mathcal{C}_{0}(r) f(r), \\
\tilde{h}(\tilde{r}) & =\frac{h(r)}{1+\mathcal{B}_{0}(r) h(r) \phi_{0}^{\prime}(r)^{2}} \\
& \times\left(1+\frac{r}{2 \mathcal{C}_{0}(r)} \frac{d \mathcal{C}_{0}(r)}{d r}\right)^{2}, \\
\tilde{\omega}(\tilde{r})-\tilde{\Omega} & =\omega(r)-\Omega,
\end{aligned}
$$

with

$$
\frac{d \mathcal{C}_{0}(r)}{d r}=-\frac{\mathcal{C}_{0 \mathcal{X}}(r) \phi_{0}^{\prime}(r)}{2}\left(h^{\prime}(r) \phi_{0}^{\prime}(r)+2 h(r) \phi_{0}^{\prime \prime}(r)\right) .
$$

In order to be compatible with the asymptotic flatness in the Jordan frame (19), we impose Eq. (16) and

$$
\begin{aligned}
\mathcal{C}_{0}(r) & \rightarrow \text { const }>0, \\
\phi_{0}^{\prime}(r)^{2} & \times \mathcal{B}_{0}(r) \rightarrow 0 \\
r h^{\prime}(r)\left(\phi_{0}^{\prime}(r)^{2}\right) \times \mathcal{C}_{0 \mathcal{X}}(r) & \rightarrow 0 \\
r \frac{d}{d r}\left(\phi_{0}^{\prime}(r)^{2}\right) \times \mathcal{C}_{0 \mathcal{X}}(r) & \rightarrow 0
\end{aligned}
$$

as $r \rightarrow \infty$. Under the conditions (32), the ADM masses (23) are shown to be invariant,

$$
\tilde{M}_{\mathrm{ADM}}=M_{\mathrm{ADM}} .
$$

Eq. (3) reduces to

$$
\begin{aligned}
T_{(m)}^{\mu \nu}= & \mathcal{C}^{3} \sqrt{1-2 \mathcal{B X}}\left[\tilde{T}_{(m)}^{\mu \nu}+\frac{1}{2} g^{\mu \alpha} g^{\nu \beta} \phi_{\alpha} \phi_{\beta}\right. \\
& \left.\times\left(\mathcal{B}, \mathcal{X} \tilde{T}_{(m)}^{\rho \sigma} \phi_{\rho} \phi_{\sigma}+\frac{\mathcal{C}_{, \mathcal{X}}}{\mathcal{C}^{2}} \tilde{T}_{(m)}\right)\right],
\end{aligned}
$$

where we have defined $\tilde{T}_{(m)}:=\tilde{g}_{\alpha \beta} \tilde{T}_{(m)}^{\alpha \beta}$. At $\mathcal{O}\left(\epsilon^{0}\right)$, namely the energy density and pressures in both the frames are related

$$
\begin{aligned}
\rho(r)= & \mathcal{C}_{0}(r)^{2} \sqrt{1+\mathcal{B}_{0}(r) h(r) \phi^{\prime}(r)^{2}} \tilde{\rho}(\tilde{r}), \\
p_{r}(r)= & \frac{\mathcal{C}_{0}(r)^{2}}{\sqrt{1+\mathcal{B}_{0}(r) h(r) \phi^{\prime}(r)^{2}}} \\
\times & {\left[\tilde{p}_{r}(\tilde{r})+\frac{h(r)\left(\phi^{\prime}(r)\right)^{2}}{2}\left(\mathcal{B}_{0 \mathcal{X}}(r) h(r) \tilde{p}_{r}(\tilde{r})\left(\phi^{\prime}(r)\right)^{2}\right.\right.} \\
& \left.\left.+\frac{\mathcal{C}_{0 \mathcal{X}}(r) \tilde{T}_{(m, 0)}(\tilde{r})}{\mathcal{C}_{0}(r)}\left(1+\mathcal{B}_{0}(r) h(r) \phi^{\prime}(r)^{2}\right)\right)\right], \\
p_{t}(r)= & \mathcal{C}_{0}(r)^{2} \sqrt{1+\mathcal{B}_{0}(r) h(r) \phi^{\prime}(r)^{2}} \tilde{p}_{t}(\tilde{r}),
\end{aligned}
$$

where $\tilde{T}_{(m, 0)}(\tilde{r})=-\tilde{\rho}(\tilde{r})+\tilde{p}_{r}(\tilde{r})+2 \tilde{p}_{t}(\tilde{r})$, and at $\mathcal{O}\left(\epsilon^{1}\right)$,

$$
\begin{aligned}
\tilde{\omega}(\tilde{r}) & =\omega(r), \\
\tilde{\Omega} & =\Omega .
\end{aligned}
$$

In general, the isotropic fluid in the Jordan frame $\tilde{p}_{r}(\tilde{r})=$ $\tilde{p}_{t}(\tilde{r})$ is mapped to the anisotropic fluid $p_{r}(r) \neq p_{t}(r)$. In general, the surface condition $\tilde{p}_{r}(\tilde{\mathcal{R}})=0$ in the Jordan frame also corresponds to the condition $p_{r}(\mathcal{R})=0$ in the Einstein frame with $\tilde{\mathcal{R}}=\tilde{\mathcal{R}}(\mathcal{R})$ for $\mathcal{C}_{0 \mathcal{X}}(\mathcal{R})=0$. The frame-dragging function and the angular velocity of the star are disformally invariant.

It is straightforward to confirm that up to $\mathcal{O}\left(\epsilon^{0}\right)$ the angular momenta and moments of inertia, Eqs. (24) and (25), respectively, are also disformally invariant

$$
\begin{aligned}
\tilde{\mathcal{J}} & =\mathcal{J}, \\
\tilde{\mathcal{I}} & =\mathcal{I} .
\end{aligned}
$$

Our results correspond to the extension of the work [20] for the subclass of Eqs. $\mathcal{C}=\mathcal{C}(\phi)$ and $\mathcal{B}=\mathcal{B}(\phi)$, which can frame the Horndeski theories [55-58]. 


\section{THE VECTOR DISFORMAL TRANSFORMATION WITHOUT THE $U(1)$ GAUGE SYMMETRY}

In this section, we consider the vector-tensor theories without the $U(1)$ gauge symmetry

$$
S=S_{g}\left[g_{\mu \nu}, A_{\mu}\right]+S_{m}\left[\tilde{g}_{\mu \nu}, \Psi_{m}\right],
$$

where the matter action is given by Eq. (2) and the relation between the two metrics is given by Eq. (11). Eq. (3) reduces to

$$
\begin{aligned}
T_{(m)}^{\mu \nu}= & \mathcal{D}(\mathcal{Y})^{3} \sqrt{1-2 \mathcal{E}(\mathcal{Y}) \mathcal{Y}}\left[\bar{T}_{(m)}^{\mu \nu}\right. \\
& \left.+g^{\mu \alpha} g^{\nu \beta} A_{\alpha} A_{\beta} \mathcal{Q}\right],
\end{aligned}
$$

where we have defined

$$
\mathcal{Q}:=\frac{\mathcal{D}, \mathcal{Y}(\mathcal{Y})}{2 \mathcal{D}(\mathcal{Y})^{2}} \tilde{T}_{(m)}+\frac{\mathcal{E}_{, \mathcal{Y}}(\mathcal{Y})}{2} \tilde{T}_{(m)}^{\rho \sigma} A_{\rho} A_{\sigma},
$$

with $\mathcal{D}, \mathcal{Y}:=\partial \mathcal{D}(Y) / \partial \mathcal{Y}$ and $\mathcal{E}_{, \mathcal{Y}}:=\partial \mathcal{E}(Y) / \partial \mathcal{Y}$. In the slow-rotation approximation, the vector field should have the following form [53]

$$
\begin{aligned}
A_{\mu} d x^{\mu} & =A_{t}(r) d t+A_{r}(r) d r+\epsilon r^{2} a_{3}(r) \sin ^{2} \theta d \varphi \\
& +\mathcal{O}\left(\epsilon^{2}\right) .
\end{aligned}
$$

In general, the metric (12) contains the components

$$
\begin{aligned}
\tilde{g}_{t r} & =\mathcal{D}_{0}(r) \mathcal{E}_{0}(r) A_{t}(r) A_{r}(r), \\
\tilde{g}_{r \varphi} & =\epsilon r^{2} a_{3}(r) A_{r}(r) \mathcal{D}_{0}(r) \mathcal{E}_{0}(r) \sin ^{2} \theta,
\end{aligned}
$$

and $\tilde{g}_{t \theta}=\tilde{g}_{\theta \varphi}=0$, where we have defined

$$
\begin{aligned}
\mathcal{D}(Y) & =\mathcal{D}_{0}(r)+\mathcal{O}\left(\epsilon^{2}\right) \\
& :=\mathcal{D}\left(\frac{1}{2 f(r)} A_{t}(r)^{2}-\frac{h(r)}{2} A_{r}(r)^{2}\right)+\mathcal{O}\left(\epsilon^{2}\right), \\
\mathcal{D}_{0 \mathcal{Y}}(r) & :=\mathcal{D}_{, \mathcal{Y}}\left(\frac{1}{2 f(r)} A_{t}(r)^{2}-\frac{h(r)}{2} A_{r}(r)^{2}\right), \\
\mathcal{E}(Y) & =\mathcal{E}_{0}(r)+\mathcal{O}\left(\epsilon^{2}\right) \\
& :=\mathcal{E}\left(\frac{1}{2 f(r)} A_{t}(r)^{2}-\frac{h(r)}{2} A_{r}(r)^{2}\right)+\mathcal{O}\left(\epsilon^{2}\right), \\
\mathcal{E}_{0 \mathcal{Y}}(r) & :=\mathcal{E}_{, \mathcal{Y}}\left(\frac{1}{2 f(r)} A_{t}(r)^{2}-\frac{h(r)}{2} A_{r}(r)^{2}\right) .
\end{aligned}
$$

In order to be compatible with the circularity conditions (17) as well as the nonzero $\mathcal{D}_{0}(r) \neq 0$, we impose either of the following two conditions separately:

- the pure conformal coupling (7);

$$
\mathcal{E}(\mathcal{Y})=0
$$

- the vanishing radial component of the vector field

$$
A_{r}(r)=0 .
$$

Relativistic star solutions with nontrivial $A_{t}(r)$ and Eq. (45) have been investigated in the models in the vectortensor theories [21-26, 59].

\section{A. The pure conformal case (44)}

First, we consider the case of Eq. (7) which relates the radial coordinates and metric components in the Jordan and Einstein frames, respectively, as

$$
\tilde{r}:=\sqrt{\mathcal{D}_{0}(r)} r
$$

and

$$
\begin{aligned}
\tilde{f}(\tilde{r}) & =\mathcal{D}_{0}(r) f(r), \\
\tilde{h}(\tilde{r}) & =h(r)\left(1+\frac{r}{2 \mathcal{D}_{0}(r)} \frac{d \mathcal{D}_{0}}{d r}(r)\right)^{2}, \\
\tilde{\omega}(\tilde{r})-\tilde{\Omega} & =\omega(r)-\Omega,
\end{aligned}
$$

where

$$
\begin{aligned}
\frac{d \mathcal{D}_{0}}{d r}(r)= & \mathcal{D}_{0 \mathcal{Y}}(r)\left[\frac{A_{t}(r)}{2 f(r)^{2}}\left(2 f(r) A_{t}^{\prime}(r)-A_{t}(r) f^{\prime}(r)\right)\right. \\
& \left.-\frac{A_{r}(r)}{2}\left(h^{\prime}(r) A_{r}(r)+2 A_{r}^{\prime}(r) h(r)\right)\right] .
\end{aligned}
$$

In order to be compatible with the asymptotic flatness in the Jordan frame (19), we impose Eq. (16) and

$$
\begin{aligned}
\mathcal{D}_{0}(r) & \rightarrow \text { const }>0, \\
r \frac{d}{d r}\left(A_{t}(r)^{2}\right) \times \mathcal{D}_{0 \mathcal{Y}}(r) & \rightarrow 0, \\
r f^{\prime}(r) A_{t}(r)^{2} \times \mathcal{D}_{0 \mathcal{Y}}(r) & \rightarrow 0, \\
r \frac{d}{d r}\left(A_{r}(r)^{2}\right) \times \mathcal{D}_{0 \mathcal{Y}}(r) & \rightarrow 0, \\
r h^{\prime}(r) A_{r}(r)^{2} \times \mathcal{D}_{0 \mathcal{Y}}(r) & \rightarrow 0,
\end{aligned}
$$

as $r \rightarrow \infty$. Under the conditions (49), the ADM masses (23) are shown to be invariant

$$
\tilde{M}_{\mathrm{ADM}}=M_{\mathrm{ADM}} \text {. }
$$

The energy-momentum tensors of matter are related via Eq. (39) with Eqs. (40) and (44). Assuming the perfect fluid form (20) and (21), respectively, comparing the frames gives, at $\mathcal{O}\left(\epsilon^{0}\right)$,

$$
\begin{aligned}
\rho(r) & =\mathcal{D}_{0}(r)^{2}\left(\tilde{\rho}(\tilde{r})+\frac{\mathcal{D}_{0 \mathcal{Y}}(r)}{2 \mathcal{D}_{0}(r)} \frac{A_{t}(r)^{2}}{f(r)} \tilde{T}(\tilde{r})\right) \\
p_{r}(r) & =\mathcal{D}_{0}(r)^{2}\left(\tilde{p}_{r}(\tilde{r})+\frac{\mathcal{D}_{0 \mathcal{Y}}(r)(r) h(r) A_{r}(r)^{2}}{2 \mathcal{D}_{0}(r)} \tilde{T}(\tilde{r})\right) \\
p_{t}(r) & =\mathcal{D}_{0}(r)^{2} \tilde{p}_{t}(\tilde{r})
\end{aligned}
$$

and at $\mathcal{O}\left(\epsilon^{1}\right)$,

$$
\begin{aligned}
\omega(r) & =\tilde{\omega}(\tilde{r}), \\
\Omega & =\tilde{\Omega}, \\
a_{3}(r) & =-\frac{\omega(r)}{f(r)} A_{t}(r) .
\end{aligned}
$$

The isotropic fluid $\tilde{p}_{r}(\tilde{r})=\tilde{p}_{t}(\tilde{r})$ in the Jordan frame can be mapped to the anisotropic fluid $p_{r}(r) \neq p_{t}(r)$ in 
the Einstein frame, unless $\mathcal{D}_{0}(r)=0$. In general, the surface condition $\tilde{p}_{r}(\tilde{\mathcal{R}})=0$ in the Jordan frame also corresponds to the condition $p_{r}(\mathcal{R})=0$ in the Einstein frame with $\tilde{\mathcal{R}}=\tilde{\mathcal{R}}(\mathcal{R})$ for $A_{r}(\mathcal{R})=0$ or $\mathcal{D}_{0 \mathcal{Y}}(\mathcal{R})=0$. Also, the frame-dragging function and the moment of inertia are conformally invariant. Rewriting the vector field $A_{\mu}$ in terms of the new radial coordinate $\tilde{r}$

$$
\begin{aligned}
A_{\mu} d x^{\mu} & =A_{t}(\tilde{r}) d t+A_{\tilde{r}}(\tilde{r}) d \tilde{r}+\epsilon \tilde{r}^{2} \tilde{a}_{3}(\tilde{r}) \sin ^{2} \theta d \varphi \\
& +\mathcal{O}\left(\epsilon^{2}\right)
\end{aligned}
$$

where

$$
\begin{aligned}
& A_{\tilde{r}}(\tilde{r}):=\frac{A_{r}(r)}{\sqrt{\mathcal{D}_{0}(r)}\left(1+\frac{r \mathcal{D}_{0, r}(r)}{2 \mathcal{D}_{0}(r)}\right)}, \\
& \tilde{a}_{3}(\tilde{r}):=\frac{a_{3}(r)}{\mathcal{D}_{0}(r)}
\end{aligned}
$$

Eq. (52c) can be rewritten as

$$
\tilde{a}_{3}(\tilde{r})=-\frac{\tilde{\omega}(\tilde{r})}{\tilde{f}(\tilde{r})} A_{t}(\tilde{r})
$$

Up to $\mathcal{O}\left(\epsilon^{1}\right)$, the relation Eq. (52c) also reads

$$
A^{\varphi}=\Omega A^{t},
$$

where $A^{\varphi}:=g^{\varphi \mu} A_{\mu}$ and $A^{t}:=g^{t \mu} A_{\mu}$, while Eq. (55) reads

$$
\tilde{A}^{\varphi}=\tilde{\Omega} \tilde{A}^{\tilde{t}},
$$

where $\tilde{A}^{\varphi}:=\tilde{g}^{\varphi \mu} A_{\mu}$ and $\tilde{A}^{t}:=\tilde{g}^{t \mu} A_{\mu}$. Thus, the azimuthal component of the vector field is induced by the rotation of the star. by

The angular momenta, defined by Eq. (24), are related

$$
\tilde{\mathcal{J}}=\mathcal{J}+\Delta \mathcal{J}_{V_{1}}
$$

where

$$
\begin{aligned}
\Delta \mathcal{J}_{V_{1}} & :=\int d r d \theta d \varphi\left(r^{2} \sin \theta \sqrt{\frac{f(r)}{h(r)}}\right) \frac{r^{2} \sin ^{2} \theta}{f(r)} \\
& \times\left(\frac{1}{2} \mathcal{D}_{0}(r) \mathcal{D}_{0 \mathcal{Y}}(r) a_{3}(r)\right) \tilde{T}_{(m)}(\tilde{r}) A_{t}(r) .
\end{aligned}
$$

The moment of inertia, defined in Eq. (25), are also not invariant

$$
\tilde{\mathcal{I}}=\mathcal{I}+\frac{\Delta \mathcal{J}_{V_{1}}}{\Omega} .
$$

The existence of the azimuthal component of the vector field causes the angular momentum and moment of inertia not to be frame-invariant.

\section{B. The case of Eq. (45)}

Next, we consider the case of Eq. (45). In the slowrotation approximation,

$$
\begin{aligned}
\mathcal{E}(Y) & =\mathcal{E}_{0}(r)+\mathcal{O}\left(\epsilon^{2}\right):=\mathcal{E}\left(\frac{A_{t}(t)^{2}}{2 f(r)}\right)+\mathcal{O}\left(\epsilon^{2}\right), \\
\mathcal{E}_{0 \mathcal{Y}}(r) & :=\mathcal{E}_{, \mathcal{Y}}\left(\frac{A_{t}(r)^{2}}{2 f(r)}\right) .
\end{aligned}
$$

The disformal tranformation (11) relates the radial coordinates and the metric components in the Jordan and Einstein frames, respectively, as Eq. (46) and

$$
\begin{aligned}
\tilde{f}(\tilde{r}) & =\mathcal{D}_{0}(r)\left(f(r)-\mathcal{E}_{0}(r) A_{t}(r)^{2}\right), \\
\tilde{h}(\tilde{r}) & =h(r)\left(1+\frac{r}{2 \mathcal{D}_{0}(r)} \frac{d \mathcal{D}_{0}}{d r}(r)\right)^{2}, \\
\tilde{\omega}(\tilde{r})-\tilde{\Omega} & =\omega(r)-\Omega+a_{3}(r) \mathcal{E}_{0}(r) A_{t}(r),
\end{aligned}
$$

where

$$
\frac{d \mathcal{D}_{0}(r)}{d r}=-\frac{\mathcal{D}_{0 \mathcal{Y}}(r) A_{t}(r)\left(-2 f(r) A_{t}^{\prime}(r)+A_{t}(r) f^{\prime}(r)\right)}{2 f(r)^{2}} .
$$

In order to be compatible with the asymptotic flatness in the Jordan frame (19), we impose Eq. (16) and

$$
\begin{aligned}
\mathcal{D}_{0}(r) & \rightarrow \text { const }>0, \\
A_{t}(r)^{2} \times \mathcal{E}_{0}(r) & \rightarrow 0, \\
r \frac{d}{d r}\left(A_{t}(r)^{2}\right) \times \mathcal{D}_{0 \mathcal{Y}}(r) & \rightarrow 0, \\
r f^{\prime}(r) A_{t}(r)^{2} \times \mathcal{D}_{0 \mathcal{Y}}(r) & \rightarrow 0, \\
a_{3}(r) A_{t}(r) \times \mathcal{E}_{0}(r) & \rightarrow 0,
\end{aligned}
$$

as $r \rightarrow \infty$. Under the conditions (64), the ADM masses (23) are shown to be invariant

$$
\tilde{M}_{\mathrm{ADM}}=M_{\mathrm{ADM}} .
$$

Comparing frames gives, at $\mathcal{O}\left(\epsilon^{0}\right)$,

$$
\begin{aligned}
\rho(r) & =\mathcal{D}_{0}(r)^{2} \sqrt{1-\frac{\mathcal{E}_{0}(r)}{f(r)} A_{t}(r)^{2}} \\
& \times\left(\frac{\tilde{\rho}}{1-\frac{\mathcal{E}_{0}(r)}{f(r)} A_{t}(r)^{2}}\right. \\
& \left.+\frac{\mathcal{D}_{0}(r) A_{t}(r)^{2}}{f(r)} \mathcal{Q}_{0}(r)\right), \\
p_{r}(r) & =\mathcal{D}_{0}(r)^{2} \sqrt{1-\frac{\mathcal{E}_{0}(r)}{f(r)} A_{t}(r)^{2}} \tilde{p}_{r}(\tilde{r}), \\
p_{t}(r) & =\mathcal{D}_{0}(r)^{2} \sqrt{1-\frac{\mathcal{E}_{0}(r)}{f(r)} A_{t}(r)^{2}} \tilde{p}_{t}(\tilde{r}),
\end{aligned}
$$


where $\mathcal{Q}_{0}(r)$ is the $\mathcal{O}\left(\epsilon^{0}\right)$ part of $\mathcal{Q}$ defined in Eq. (40),

$$
\mathcal{Q}_{0}(r):=\frac{\mathcal{E}_{0 \mathcal{Y}}(r) A_{t}(r)^{2} \tilde{\rho}(\tilde{r})}{2 f(r)\left(1-\frac{\mathcal{E}_{0}(r) A_{t}(r)^{2}}{f(r)}\right)}+\frac{\mathcal{D}_{0 \mathcal{Y}}(r) \tilde{T}_{(m)}(\tilde{r})}{2 \mathcal{D}_{0}(r)^{2}},
$$

and at $\mathcal{O}\left(\epsilon^{1}\right)$,

$$
\begin{aligned}
\omega(r) & =\frac{\tilde{\omega}(\tilde{r})}{1-\frac{\mathcal{E}_{0}(r)}{f(r)} A_{t}(r)^{2}}, \\
\Omega & =\tilde{\Omega}, \\
a_{3}(r) & =-\frac{\omega(r)}{f(r)} A_{t}(r) .
\end{aligned}
$$

In general, the surface condition $\tilde{p}_{r}(\tilde{\mathcal{R}})=0$ in the Jordan frame also corresponds to the condition $p_{r}(\mathcal{R})=0$ in the Einstein frame with $\tilde{\mathcal{R}}=\tilde{\mathcal{R}}(\mathcal{R})$. The frame-dragging function and the moment of inertia are conformally invariant, and the last term represents the induced magnetic component of the vector field induced by the rotation of the spacetime. The vector field $A_{\mu}$ in terms of the new radial coordinate $\tilde{r}$ can be expressed as Eq. (53) with

$$
\begin{aligned}
& A_{\tilde{r}}(\tilde{r})=0, \\
& \tilde{a}_{3}(\tilde{r})=\frac{a_{3}(r)}{\mathcal{D}_{0}(r)},
\end{aligned}
$$

and then Eq. (68c) is identical to Eq. (52c) and can be rewritten as Eq. (55). Up to $\mathcal{O}\left(\epsilon^{1}\right)$, the relation Eq. (68c) also reads Eq. (56), while Eq. (55) reads Eq. (57). Thus, the azimuthal component of the vector field is induced from the time component of it by the rotation of the star.

The angular momenta and moment of inertia defined by Eqs. (24) and (25), respectively, are related by

$$
\begin{aligned}
& \tilde{\mathcal{J}}=\mathcal{J}+\Delta \mathcal{J}_{V_{2}}, \\
& \tilde{\mathcal{I}}=\mathcal{I}+\frac{\Delta \mathcal{J}_{V_{2}}}{\Omega},
\end{aligned}
$$

where the correction term is given by

$$
\begin{aligned}
\Delta \mathcal{J}_{V_{2}} & :=\int d r d \theta d \varphi\left(r^{2} \sin \theta \sqrt{\frac{f(r)}{h(r)}}\right) \\
& \times \frac{r^{2} \mathcal{D}_{0}(r)^{2} A_{t}(r)}{f(r)} a_{3}(r) \sqrt{1-\frac{\mathcal{E}_{0}(r) A_{t}(r)^{2}}{f(r)}} \sin ^{2} \theta \\
& \times\left(\frac{\mathcal{E}_{0}(r) \hat{\rho}(\tilde{r})}{1-\frac{\mathcal{E}_{0}(r)}{f(r)} A_{t}(r)^{2}}\left(1+\frac{\mathcal{E}_{0 \mathcal{Y}}(r) \mathcal{D}_{0}(r) A_{t}(r)^{2}}{2 \mathcal{E}_{0}(r) f(r)}\right)\right. \\
& \left.+\frac{\mathcal{D}_{0 \mathcal{Y}}(r)}{2 \mathcal{D}_{0}(r)} \tilde{T}_{(m)}(\tilde{r})\right) .
\end{aligned}
$$

The existence of the azimuthal component of the vector field causes the angular momentum and moment of inertia not to be frame-invariant.

\section{THE VECTOR DISFORMAL TRANSFORMATION WITH THE $U(1)$ GAUGE SYMMETRY}

Finally, we consider the vector-tensor theories with the $U(1)$ gauge symmetry

$$
S=S_{g}\left[g_{\mu \nu}, F_{\mu \nu}\right]+S_{m}\left[\tilde{g}_{\mu \nu}, \Psi_{m}\right],
$$

where the matter action is given by Eq. (2) and the relation between the two metrics is given by Eq. (12). Up to $\mathcal{O}\left(\epsilon^{1}\right)$, the vector field is assumed to be

$$
A_{\mu} d x^{\mu}=A_{t}(r) d t+\epsilon r^{2} a_{3}(r) \sin ^{2} \theta d \varphi+\mathcal{O}\left(\epsilon^{2}\right),
$$

where $A_{t}(r)$ and $a_{3}(r)$ are the functions of $r$. Without loss of generality, we may set $A_{r}(r)=0$ because of the $U(1)$ gauge symmetry.

We expand $\mathcal{F}$ and $\mathcal{G}$ in terms of $\epsilon$,

$$
\begin{aligned}
& \mathcal{F}=\mathcal{F}_{0}(r)+\mathcal{O}\left(\epsilon^{2}\right):=-\frac{2 h(r) A_{t}^{\prime}(r)^{2}}{f(r)}+\mathcal{O}\left(\epsilon^{2}\right), \\
& \mathcal{G}=\mathcal{G}_{0}(r)+\mathcal{O}\left(\epsilon^{2}\right):=\frac{2 h(r)^{2} A_{t}^{\prime}(r)^{4}}{f(r)^{2}}+\mathcal{O}\left(\epsilon^{2}\right),
\end{aligned}
$$

and define

$$
\begin{aligned}
& \mathcal{V}_{0}(r)=\mathcal{V}\left(\mathcal{F}_{0}(r), \mathcal{G}_{0}(r)\right), \\
& \mathcal{W}_{0}(r)=\mathcal{W}\left(\mathcal{F}_{0}(r), \mathcal{G}_{0}(r)\right), \\
& \mathcal{V}_{0 \mathcal{F}}(r)=\mathcal{V}_{, \mathcal{F}}\left(\mathcal{F}_{0}(r), \mathcal{G}_{0}(r)\right), \\
& \mathcal{W}_{0 \mathcal{F}}(r)=\mathcal{W}_{, \mathcal{F}}\left(\mathcal{F}_{0}(r), \mathcal{G}_{0}(r)\right), \\
& \mathcal{V}_{0 \mathcal{G}}(r)=\mathcal{V}_{, \mathcal{G}}\left(\mathcal{F}_{0}(r), \mathcal{G}_{0}(r)\right), \\
& \mathcal{W}_{0 \mathcal{G}}(r)=\mathcal{W}_{, \mathcal{G}}\left(\mathcal{F}_{0}(r), \mathcal{G}_{0}(r)\right),
\end{aligned}
$$

as the $\mathcal{O}\left(\epsilon^{0}\right)$ part of $\mathcal{V}, \mathcal{W}, \mathcal{V}_{, \mathcal{F}}:=\partial \mathcal{V} / \partial \mathcal{F}, \mathcal{V}_{\mathcal{G}}:=\partial \mathcal{V} / \partial \mathcal{G}$, $\mathcal{W}_{, \mathcal{F}}:=\partial \mathcal{W} / \partial \mathcal{F}$, and $\mathcal{W}_{\mathcal{G}}:=\partial \mathcal{W} / \partial \mathcal{G}$. Eq. (12) relates the radial coordinates and the metric components in the Jordan and Einstein frames, respectively, by

$$
\tilde{r}:=\sqrt{\mathcal{V}_{0}(r)} r,
$$

and

$$
\tilde{f}(\tilde{r})=\mathcal{V}_{0}(r) f(r)\left(1-\frac{h(r) \mathcal{W}_{0}(r)}{f(r)}\left(A_{t}^{\prime}(r)\right)^{2}\right),
$$

$$
\begin{aligned}
& \tilde{h}(\tilde{r})=\frac{\left(1+\frac{r}{2 \mathcal{V}_{0}(r)} \frac{d \mathcal{V}_{0}}{d r}(r)\right)^{2}}{1-\frac{h(r) \mathcal{W}_{0}(r)}{f(r)}\left(A_{t}^{\prime}(r)\right)^{2}} h(r), \\
& \tilde{\omega}(\tilde{r})-\tilde{\Omega}=\omega(r)-\Omega \\
& +\frac{h(r) A_{t}^{\prime}(r) \mathcal{W}_{0}(r)}{r^{2}} \frac{d}{d r}\left(r^{2} a_{3}(r)\right),
\end{aligned}
$$

where

$$
\begin{aligned}
\frac{d \mathcal{V}_{0}}{d r}(r) & =-\frac{2 A_{t}^{\prime}(r)}{f(r)^{2}} \\
& \times\left[A_{t}^{\prime}(r)\left(-h(r) f^{\prime}(r)+f(r) h^{\prime}(r)\right)\right. \\
& \left.+2 f(r) h(r) A_{t}^{\prime \prime}(r)\right] \\
& \times\left[\mathcal{V}_{0 \mathcal{F}}(r)-\frac{2 h(r) A_{t}^{\prime}(r)^{2}}{f(r)} \mathcal{V}_{0 \mathcal{G}}(r)\right] .
\end{aligned}
$$


In order to be compatible with the asymptotic flatness in the Jordan frame (19), we impose Eq. (16) and

$$
\begin{aligned}
\mathcal{V}_{0}(r) & \rightarrow \text { const }>0, \\
\left(A_{t}^{\prime}(r)\right)^{2} \times \mathcal{W}_{0}(r) & \rightarrow 0, \\
r f^{\prime}(r)\left(A_{t}^{\prime}(r)\right)^{2} \times \mathcal{V}_{0 \mathcal{F}}(r) & \rightarrow 0, \\
r h^{\prime}(r)\left(A_{t}^{\prime}(r)\right)^{2} \times \mathcal{V}_{0 \mathcal{F}}(r) & \rightarrow 0, \\
r \frac{d}{d r}\left[\left(A_{t}^{\prime}(r)\right)^{2}\right] \times \mathcal{V}_{0 \mathcal{F}}(r) & \rightarrow 0, \\
r f^{\prime}(r)\left(A_{t}^{\prime}(r)\right)^{4} \times \mathcal{V}_{0 \mathcal{G}}(r) & \rightarrow 0, \\
r h^{\prime}(r)\left(A_{t}^{\prime}(r)\right)^{4} \times \mathcal{V}_{0 \mathcal{G}}(r) & \rightarrow 0, \\
r \frac{d}{d r}\left[\left(A_{t}^{\prime}(r)\right)^{4}\right] \times \mathcal{V}_{0 \mathcal{G}}(r) & \rightarrow 0, \\
\frac{A_{t}^{\prime}(r)}{r^{2}} \frac{d}{d r}\left[r^{2} a_{3}(r)\right] \times \mathcal{W}_{0}(r) & \rightarrow 0,
\end{aligned}
$$

as $r \rightarrow \infty$. Under the conditions (79), the ADM masses
(23) are shown to be invariant,

$$
\tilde{M}_{\mathrm{ADM}}=M_{\mathrm{ADM}} \text {. }
$$

Eq. (3) reduces to

$$
\begin{aligned}
T_{(m)}^{\mu \nu} & =\mathcal{V} \sqrt{\frac{\tilde{g}}{g}}\left[\tilde{T}_{(m)}^{\mu \nu}-2 \mathcal{Q}_{\mathcal{F}} g^{\mu \alpha} g^{\nu \beta} g^{\rho \sigma} F_{\alpha \rho} F_{\beta \sigma}\right. \\
& -4 \mathcal{Q}_{\mathcal{G}} g^{\mu \kappa} g^{\nu \omega} g^{\alpha \beta} g^{\rho \sigma} g^{\gamma \delta} F_{\kappa \alpha} F_{\rho \beta} F_{\sigma \gamma} F_{\omega \delta} \\
& \left.-\mathcal{W} g^{\mu \kappa} g^{\nu \omega} F_{\kappa \alpha} F_{\omega \beta} \tilde{T}_{(m)}^{\alpha \beta}\right]
\end{aligned}
$$

where

$$
\begin{aligned}
\mathcal{Q}_{\mathcal{F}} & :=\frac{\mathcal{V}_{, \mathcal{F}}}{\mathcal{V}^{2}} \tilde{T}_{(m)}+\mathcal{W}_{, \mathcal{F}} g^{\rho \sigma} F_{\rho \alpha} F_{\sigma \beta} \tilde{T}_{(m)}^{\alpha \beta}, \\
\mathcal{Q}_{\mathcal{G}} & :=\frac{\mathcal{V}_{, \mathcal{G}}}{\mathcal{V}^{2}} \tilde{T}_{(m)}+\mathcal{W}_{, \mathcal{G}} g^{\rho \sigma} F_{\rho \alpha} F_{\sigma \beta} \tilde{T}_{(m)}^{\alpha \beta} .
\end{aligned}
$$

Comparing frames gives, at $\mathcal{O}\left(\epsilon^{0}\right)$,

$$
\begin{aligned}
& \rho(r)=\mathcal{V}_{0}(r)\left[\left.\sqrt{\frac{\tilde{g}}{g}}\right|_{0}(r)\right]\left\{\frac{\tilde{\rho}(\tilde{r})-\frac{\mathcal{W}_{0}(r) h(r)\left(A_{t}^{\prime}\right)^{2}}{f(r)} \tilde{p}_{r}(\tilde{r})}{\mathcal{V}_{0}(r)\left(1-\frac{h(r) \mathcal{W}_{0}(r)}{f(r)}\left(A_{t}^{\prime}(r)\right)^{2}\right)}-2\left(\frac{\mathcal{Q}_{0 \mathcal{F}}(r) h(r)\left(A_{t}^{\prime}(r)\right)^{2}}{f(r)}-\frac{2 \mathcal{Q}_{0 \mathcal{G}}(r) h(r)^{2}\left(A_{t}^{\prime}(r)\right)^{4}}{f(r)^{2}}\right)\right\}, \\
& p_{r}(r)=\mathcal{V}_{0}(r)\left[\left.\sqrt{\frac{\tilde{g}}{g}}\right|_{0}(r)\right]\left\{\frac{\tilde{p}_{r}(\tilde{r})-\frac{\mathcal{W}_{0}(r) h(r)\left(A_{t}^{\prime}(r)\right)^{2}}{f(r)} \tilde{\rho}(\tilde{r})}{\mathcal{V}_{0}(r)\left(1-\frac{h(r) \mathcal{W}_{0}(r)}{f(r)}\left(\left(A_{t}^{\prime}(r)\right)^{2}\right)\right.}+2\left(\frac{\mathcal{Q}_{0 \mathcal{F}}(r) h(r)\left(A_{t}^{\prime}(r)\right)^{2}}{f(r)}-\frac{2 \mathcal{Q}_{0 \mathcal{G}}(r) h(r)^{2}\left(A_{t}^{\prime}(r)\right)^{4}}{f(r)^{2}}\right)\right\}, \\
& p_{t}(r)=\left[\left.\sqrt{\frac{\tilde{g}}{g}}\right|_{0} ^{(}(r)\right] \tilde{p}_{t}(\tilde{r}),
\end{aligned}
$$

where

$$
\begin{aligned}
\left.\sqrt{\frac{\tilde{g}}{g}}\right|_{0}(r) & :=\mathcal{V}_{0}(r)^{2}\left(1-\frac{h(r) \mathcal{W}_{0}(r)}{f(r)}\left(A_{t}^{\prime}(r)\right)^{2}\right) \\
\mathcal{Q}_{0 \mathcal{F}}(r) & :=\frac{\mathcal{V}_{0 \mathcal{F}}(r)}{\mathcal{V}_{0}(r)^{2}} \tilde{T}_{(m)}+\mathcal{W}_{0 \mathcal{F}}(r) h(r) A_{t}^{\prime}(r)^{2} \\
& \times \frac{\tilde{\rho}(\tilde{r})-\tilde{p}_{r}(\tilde{r})}{\mathcal{V}_{0} f(r)\left(1-\frac{h(r) \mathcal{W}_{0}(r)}{f(r)} A_{t}^{\prime}(r)^{2}\right)}, \\
\mathcal{Q}_{0 \mathcal{G}}(r) & :=\frac{\mathcal{V}_{0 \mathcal{G}}(r)}{\mathcal{V}_{0}(r)^{2}} \tilde{T}_{(m)}+\mathcal{W}_{0 \mathcal{G}}(r) h(r) A_{t}^{\prime}(r)^{2} \\
& \times \frac{\tilde{\rho}(\tilde{r})-\tilde{p}_{r}(\tilde{r})}{\mathcal{V}_{0}(r) f(r)\left(1-\frac{h(r) \mathcal{W}_{0}(r)}{f(r)} A_{t}^{\prime}(r)^{2}\right)},
\end{aligned}
$$

and at $\mathcal{O}\left(\epsilon^{1}\right)$,

$$
\begin{aligned}
& \omega(r)=\frac{\tilde{\omega}(r)}{1-\frac{h(r) \mathcal{W}_{0}(r)}{f(r)}\left(A_{t}^{\prime}(r)\right)^{2}}, \\
& \Omega=\tilde{\Omega}, \\
& \left(r^{2} a_{3}(r)\right)_{, r}+\frac{r^{2} \omega(r)}{f(r)} A_{t}^{\prime}(r)=0 .
\end{aligned}
$$

In general, the surface condition $\tilde{p}_{r}(\tilde{\mathcal{R}})=0$ in the Jordan frame also corresponds to the condition $p_{r}(\mathcal{R})=0$ in the Einstein frame with $\tilde{\mathcal{R}}=\tilde{\mathcal{R}}(\mathcal{R})$ only for $\mathcal{W}_{0}(\mathcal{R})=$ $\mathcal{Q}_{0 \mathcal{F}}(\mathcal{R})=\mathcal{Q}_{0 \mathcal{G}}(\mathcal{R})=0$. Rewriting the vector field $A_{\mu}$ in terms of the new radial coordinate $\tilde{r}$

$$
A_{\mu} d x^{\mu}=A_{t}(\tilde{r}) d t+\epsilon \tilde{r}^{2} \tilde{a}_{3}(\tilde{r}) \sin ^{2} \theta d \varphi+\mathcal{O}\left(\epsilon^{2}\right),
$$

where

$$
\tilde{a}_{3}(\tilde{r}):=\frac{a_{3}(r)}{\mathcal{V}_{0}(r)},
$$


Eq. (85c) can be rewritten as

$$
\left(\tilde{r}^{2} \tilde{a}_{3}(r)\right)_{, \tilde{r}}+\frac{\tilde{r}^{2} \tilde{\omega}(\tilde{r})}{\tilde{f}(\tilde{r})} A_{t, \tilde{r}}(\tilde{r})=0 .
$$

Up to $\mathcal{O}\left(\epsilon^{1}\right)$, the relation Eq. (85c) also reads

$$
F_{r}^{\varphi}=\Omega F_{r}{ }^{t},
$$

where $F_{r}^{\varphi}:=g^{\varphi \mu} F_{r \mu}$ and $F_{r}{ }^{t}:=g^{t \mu} F_{r \mu}$, while Eq. (88) reads

$$
\tilde{F}_{\tilde{r}}^{\varphi}=\tilde{\Omega} \tilde{F}_{\tilde{r}}^{t},
$$

where $\tilde{F}_{\tilde{r}}^{\varphi}:=\tilde{g}^{\varphi \mu} F_{\tilde{r} \mu}$ and $\tilde{F}_{\tilde{r}}^{t}:=\tilde{g}^{t \mu} F_{\tilde{r} \mu}$. Thus, the magnetic component of the vector field is induced from the electric component of it by the rotation of the star.

The angular momenta and moment of inertia defined by Eqs. (24) and (25), respectively, are related by

$$
\begin{aligned}
& \tilde{\mathcal{J}}=\mathcal{J}+\Delta \mathcal{J}_{F}, \\
& \tilde{\mathcal{I}}=\mathcal{I}+\frac{\Delta \mathcal{J}_{F}}{\Omega},
\end{aligned}
$$

where

$$
\begin{aligned}
\Delta \mathcal{J}_{F} & :=\int d r d \theta d \varphi\left(r^{2} \sin \theta \sqrt{\frac{f(r)}{h(r)}}\right) \\
& \times \frac{d}{d r}\left(r^{2} a_{3}(r)\right)\left(\mathcal{V}_{0}(r)^{2} A_{t}^{\prime}(r) \sin ^{2} \theta\right) \\
& \times\left[\frac{\mathcal{W}_{0}(r) h(r)}{f(r)}\left(\tilde{\rho}(\tilde{r})-\tilde{p}_{r}(\tilde{r})\right)\right. \\
& -2 \mathcal{V}_{0}(r)\left(\mathcal{Q}_{0 \mathcal{F}}(r) \frac{h(r)}{f(r)}-2 \mathcal{Q}_{0 \mathcal{G}}(r) \frac{h(r)}{f(r)}\left(A_{t}^{\prime}(r)\right)^{2}\right) \\
& \left.\times\left(1-\frac{h(r) \mathcal{W}_{0}(r)}{f(r)}\left(A_{t}^{\prime}(r)\right)^{2}\right)\right] .
\end{aligned}
$$

Thus, the difference in the angular momenta and moments of inertia is given by the magnetic component of the vector field $F_{r \varphi}$. The existence of the magnetic component of the vector field strength causes the angular momentum and moment of inertia not to be frame-invariant.

\section{CONCLUSIONS}

In this paper, we have investigated how physical quantities associated with relativistic stars in the Jordan and Einstein frames are related by the generalized disformal transformations constructed by the scalar and vector fields. We have considered the rigidly rotating star within the slow-rotation approximation, and expanded the spacetime metric and the scalar or vector field in terms of the small dimensionless parameter which is of the order of the dimensionless spin of the star. We have considered the most general scalar disformal transformation constructed by the scalar field (10), and by the vector field without and with the $U(1)$ gauge symmetry, Eqs. (11) and (12), respectively.
We have called the metric after the disformal transformation $\tilde{g}_{\mu \nu}$ the Jordan frame, since in this frame the scalar field was not directly coupled to the metric. as in Eq. (2). Correspondingly, we called the metric before the disformal transformation $g_{\mu \nu}$ the Einstein frame. The relation of the radial coordinates and the metric functions could be found via the direct comparisons of the Jordan and Einstein frames. Similarly, the relation of the components of the matter energy-momentum tensor could be found through Eq. (3). We have required that the metric in the Jordan frame $\tilde{g}_{\mu \nu}$ satisfies the circularity conditions (17), which in the slow-rotation limit reduced to the form of Eq. (18). The circularity conditions could be automatically satisfied in the case of the scalar disformal transformation (10) and the vector disformal transformation with the $U(1)$ symmetry (12). On the other hand, in the case of the vector disformal transformation without the $U(1)$ gauge symmetry, Eq. (11), the circularity conditions could be satisfied in the case of either the pure conformal coupling or the vanishing radial component of the vector field.

At the zeroth order of the slow-rotation approximation, by imposing that the spacetime metrics $g_{\mu \nu}$ and $\tilde{g}_{\mu \nu}$ in the Einstein and Jordan frames were asymptotically flat, we have obtained the conditions that the metric functions and the scalar or vector field had to be satisfied at the spatial infinity. With these conditions, we have shown that the ADM mass is frame-invariant.

At the first order of the slow-rotation approximation, we have discussed the disformal transformation of the physical quantities associated with the rotation of the star, namely, the frame-dragging function, angular velocity, angular momentum, and moment of inertia of the star. We have shown that the angular velocity of the star is frame-invariant in all the classes of the disformal transformation. On the other hand, while the angular momentum and moment of inertia were invariant under the scalar disformal transformation (10), they were not invariant under the vector disformal transformation without and with the $U(1)$ gauge symmetry, such as Eqs. (11) and (12), respectively. Apart from the relations of the above quantities, we have also obtained the relation between the frame-dragging function and the azimuthal component of the vector field (the magnetic component of the vector field strength) without (with) the $U(1)$ gauge symmetry, Eq. (52c) (Eq. (85c)). These relations could be interpreted as the induction of the azimuthal component of the vector field (the magnetic component of the vector field strength) without (with) the $U(1)$ gauge symmetry, by the slow rotation of the star, Eq. (56) (Eq. (89)).

It would be important to extend our analysis to the second-order of the slow rotation approximation, which should cover the subjects of the tidal deformability and universal relations [60-64]. It would also be interesting to explore our analysis to the more general class of the disformal transformation constructed by e.g., higher-order derivatives, other field species, and multiple components 
of the fields. Definitively, these subjects would require more computations and should be left for our future publications.

\section{ACKNOWLEDGMENTS}

M.M. was supported by the Portuguese national fund through the Fundação para a Ciência e a Tecnologia in the scope of the framework of the Decree-Law $57 / 2016$ of August 29, changed by Law $57 / 2017$ of July 19, and the CENTRA through the Project No. UIDB/00099/2020.
[1] C. M. Will, Living Rev. Rel. 17, 4 (2014), arXiv:1403.7377 [gr-qc].

[2] E. Berti et al., Class. Quant. Grav. 32, 243001 (2015), arXiv:1501.07274 [gr-qc].

[3] E. Berti, K. Yagi, H. Yang, and N. Yunes, Gen. Rel. Grav. 50, 49 (2018), arXiv:1801.03587 [gr-qc].

[4] E. Berti, K. Yagi, and N. Yunes, Gen. Rel. Grav. 50, 46 (2018), arXiv:1801.03208 [gr-qc].

[5] D. Psaltis et al. (EHT), Phys. Rev. Lett. 125, 141104 (2020), arXiv:2010.01055 [gr-qc].

[6] D. Psaltis, Gen. Rel. Grav. 51, 137 (2019), arXiv:1806.09740 [astro-ph.HE].

[7] D. Psaltis, D. Perrodin, K. R. Dienes, and I. Mocioiu, Phys.Rev.Lett. 100, 091101 (2008), arXiv:0710.4564 [astro-ph].

[8] H. Motohashi and M. Minamitsuji, Phys. Lett. B781, 728 (2018), arXiv:1804.01731 [gr-qc].

[9] J. M. Lattimer and M. Prakash, Phys.Rept. 442, 109 (2007), arXiv:astro-ph/0612440 [astro-ph].

[10] F. Özel and P. Freire, Ann. Rev. Astron. Astrophys. 54, 401 (2016), arXiv:1603.02698 [astro-ph.HE].

[11] T. Damour and G. Esposito-Farèse, Phys.Rev.Lett. 70, 2220 (1993).

[12] T. Damour and G. EspositoFarèse, Phys.Rev. D54, 1474 (1996), arXiv:gr-qc/9602056 [gr-qc].

[13] T. Harada, Prog.Theor.Phys. 98, 359 (1997), arXiv:gr-qc/9706014 [gr-qc].

[14] T. Harada, Phys.Rev. D57, 4802 (1998), arXiv:gr-qc/9801049 [gr-qc].

[15] J. Novak, Phys.Rev. D58, 064019 (1998), arXiv:gr-qc/9806022 [gr-qc].

[16] C. Palenzuela, E. Barausse, M. Ponce, and L. Lehner, Phys.Rev. D89, 044024 (2014), arXiv:1310.4481 [gr-qc].

[17] L. Sampson, N. Yunes, N. Cornish, M. Ponce, E. Barausse, A. Klein, C. Palenzuela, and L. Lehner, Phys. Rev. D 90, 124091 (2014), arXiv:1407.7038 [gr-qc].

[18] P. Pani and E. Berti, Phys.Rev. D90, 024025 (2014), arXiv:1405.4547 [gr-qc].

[19] H. O. Silva, C. F. B. Macedo, E. Berti, and L. C. B. Crispino, Class. Quant. Grav. 32, 145008 (2015), arXiv:1411.6286 [gr-qc].

[20] M. Minamitsuji and H. O. Silva, Phys. Rev. D93, 124041 (2016), arXiv:1604.07742 [gr-qc].

[21] F. M. Ramazanoğlu, Phys. Rev. D96, 064009 (2017), arXiv:1706.01056 [gr-qc].

[22] F. M. Ramazanoğlu, Phys. Rev. D99, 084015 (2019),
arXiv:1901.10009 [gr-qc].

[23] L. Annulli, V. Cardoso, and L. Gualtieri, Phys. Rev. D99, 044038 (2019), arXiv:1901.02461 [gr-qc].

[24] F. M. Ramazanoğlu and K. İ. Ünlütürk, $\quad$ Phys. Rev. D100, 084026 (2019), arXiv:1910.02801 [gr-qc].

[25] R. Kase, M. Minamitsuji, and S. Tsujikawa, Phys. Rev. D 102, 024067 (2020), arXiv:2001.10701 [gr-qc].

[26] M. Minamitsuji, Phys. Rev. D 101, 104044 (2020), arXiv:2003.11885 [gr-qc].

[27] L. Heisenberg, arXiv:1402.7026 [hep-th].

JCAP 05, 015 (2014),

[28] A. De Felice, L. Heisenberg, R. Kase, S. Tsujikawa, Y.-l. Zhang, and G.-B. Zhao, Phys.Rev.D 93, 104016 (2016), arXiv:1602.00371 [gr-qc].

[29] F. M. Ramazano glu, Phys. Rev. D 98, 044011 (2018), [Erratum: Phys.Rev.D 100, 029903 (2019)], arXiv:1804.00594 [gr-qc].

[30] N. Makino and M. Sasaki, Prog. Theor. Phys. 86, 103 (1991).

[31] J.-O. Gong, J.-c. Hwang, W.-I. Park, M. Sasaki, and Y.-S. Song, JCAP 1109, 023 (2011), arXiv:1107.1840 [gr-qc].

[32] T. Chiba and M. Yamaguchi, JCAP 0810, 021 (2008), arXiv:0807.4965 [astro-ph].

[33] T. Chiba and M. Yamaguchi, JCAP 10, 040 (2013), arXiv:1308.1142 [gr-qc].

[34] M. Minamitsuji, Phys. Lett. B737, 139 (2014), arXiv:1409.1566 [astro-ph.CO].

[35] S. Tsujikawa, JCAP 1504, 043 (2015), arXiv:1412.6210 [hep-th].

[36] Y. Watanabe, A. Naruko, and M. Sasaki, Europhys. Lett. 111, 39002 (2015), arXiv:1504.00672 [gr-qc].

[37] H. Motohashi and J. White, JCAP 1602, 065 (2016), arXiv:1504.00846 [gr-qc].

[38] T. Chiba, F. Chibana, and M. Yamaguchi, JCAP 06, 003 (2020), arXiv:2003.10633 [gr-qc].

[39] J. B. Hartle, Astrophys. J. 150, 1005 (1967).

[40] J. B. Hartle and K. S. Thorne, Astrophys.J. 153, 807 (1968).

[41] D. Langlois and K. Noui, JCAP 1602, 034 (2016), arXiv:1510.06930 [gr-qc].

[42] J. Ben Achour, D. Langlois, and K. Noui, Phys. Rev. D93, 124005 (2016), arXiv:1602.08398 [gr-qc].

[43] J. Ben Achour, M. Crisostomi, K. Koyama, D. Langlois, K. Noui, and G. Tasinato, JHEP 12, 100 (2016), arXiv:1608.08135 [hep-th]. 
[44] D. Langlois, Int. J. Mod. Phys. D 28, 1942006 (2019), arXiv:1811.06271 [gr-qc].

[45] R. P. Woodard, Scholarpedia 10, 32243 (2015), arXiv:1506.02210 [hep-th].

[46] G. Tasinato,

JHEP 04, 067 (2014), arXiv:1402.6450 [hep-th].

[47] R. Kimura, A. Naruko, and D. Yoshida, JCAP 1701, 002 (2017), arXiv:1608.07066 [gr-qc].

[48] A. E. Gumrukcuoglu and R. Namba, Phys. Rev. D 100, 124064 (2019), arXiv:1907.12292 [hep-th].

[49] A. De Felice and A. Naruko, Phys. Rev. D 101, 084044 (2020), arXiv:1911.10960 [gr-qc].

[50] W. Kundt and M. Trumper, Z. Phys. 192, 419 (1966).

[51] B. Carter, J. Math. Phys. 10, 70 (1969).

[52] R. M. Wald, General Relativity (University of Chicago Press, Chicago, 1984).

[53] M. Minamitsuji, Phys. Rev. D 102, 124017 (2020), arXiv:2012.13526 [gr-qc].

[54] Y. Xie, J. Zhang, H. O. Silva, C. de Rham, H. Witek, and N. Yunes, (2021), arXiv:2103.03925 [gr-qc].

[55] G. W. Horndeski, Int.J.Theor.Phys. 10, 363 (1974).

[56] A. Nicolis, R. Rattazzi, and
E. Trincherini, Phys.Rev. D79, 064036 (2009), arXiv:0811.2197 [hep-th].

[57] C. Deffayet, S. Deser, and G. EspositoFarèse, Phys. Rev. D80, 064015 (2009), arXiv:0906.1967 [gr-qc].

[58] T. Kobayashi, M. Yamaguchi, and J. Yokoyama, Prog. Theor. Phys. 126, 511 (2011), arXiv:1105.5723 [hep-th].

[59] R. Kase, M. Minamitsuji, and S. Tsujikawa, Phys. Rev. D97, 084009 (2018), arXiv:1711.08713 [gr-qc].

[60] K. Yagi and N. Yunes, Science 341, 365 (2013), arXiv:1302.4499 [gr-qc].

[61] G. Martinon, A. Maselli, L. Gualtieri, and V. Ferrari, Phys.Rev. D90, 064026 (2014), arXiv:1406.7661 [gr-qc].

[62] C. Breu and L. Rezzolla, Mon. Not. Roy. Astron. Soc. 459, 646 (2016), arXiv:1601.06083 [gr-qc].

[63] K. Yagi and N. Yunes, Phys. Rept. 681, 1 (2017), arXiv:1608.02582 [gr-qc].

[64] D. D. Doneva and G. Pappas, Astrophys. Space Sci. Libr. 457, 737 (2018), arXiv:1709.08046 [gr-qc]. 